Mots. Les langages du politique

\title{
Des couleurs et de la politique
}

Valérie Bonnet, Hugues Constantin de Chanay et Dominique DESMARCHELIER

\section{Q OpenEdition}

Journals

Édition électronique

URL : https://journals.openedition.org/mots/21664

DOI : $10.4000 /$ mots. 21664

ISSN : 1960-6001

\section{Éditeur}

ENS Éditions

\section{Édition imprimée}

Date de publication : 15 septembre 2014

Pagination : 5-11

ISBN : 978-2-84788-543-9

ISSN : 0243-6450

\section{Référence électronique}

Valérie Bonnet, Hugues Constantin de Chanay et Dominique DESMARCHELIER, « Des couleurs et de la politique », Mots. Les langages du politique [En ligne], 105 | 2014, mis en ligne le 15 septembre 2014, consulté le 23 avril 2022. URL : http://journals.openedition.org/mots/21664 ; DOI : https://doi.org/ $10.4000 /$ mots. 21664 


\section{Valérie Bonnet, Hugues Constantin de Chanay, Dominique Desmarchelier}

\section{Des couleurs et de la politique}

Lorsque nous avons eu l'idée de consacrer une réflexion d'ensemble au rôle joué par les termes de couleurs dans le discours politique, nous avions en tête une double opportunité scientifique :

- d'un point de vue strictement lexicologique, ces termes qui proposent à toute tentative de classement une grille perceptuelle d'apparence discrète (au sens structural - tout se passe comme si, à l'instar de ce que proposent les termes de couleurs, le spectre des couleurs était lui-même partitionné, réparti selon des seuils qui paraissent appartenir au champ du réel mais peuvent émaner aussi de la structure du langage) semblent paradoxalement échapper en grande partie aux descriptions réalistes et investir au contraire des domaines symboliques;

- du point de vue de l'analyse des discours politiques, leur rôle organisateur quasi universel, et ce sous des formes très diversifiées, incite à se demander dans quelle mesure, plus que de simplement exprimer des différences, ils participent à leur existence et configurent la politisation même des discours.

\section{Point de vue lexicologique}

Les termes de couleurs sont, à l'heure actuelle, l'exemple type de domaine lexical où l'analyse lexicologique doit impérativement croiser des études de discours. Ils sont traditionnellement considérés comme inanalysables en traits distinctifs. En témoignent les définitions lexicographiques dites « extensionnelles», c'est-àdire qu'elles font massivement appel à l'exemple : «Qui est d'une couleur dont la nature offre de nombreux exemples, comme un ciel sans nuages, certaines fleurs (le bleuet), le saphir» (définition du Petit Robert pour l'adjectif bleu).

L'exemple est parfois étayé d'un appel discret à la catégorisation scientifique lorsque la langue l'entérine par ailleurs (infrarouge, ultraviolet) : «Qui est de

Université Paul Sabatier Toulouse 3, LERASS

valerie.bonnet@free.fr

Université Lyon 2, ICAR (UMR 5191, CNRS)

huguesconstantindechanay@yahoo.fr

CEDITEC

d_desmarchelier@orange.fr

Mots. Les langages du politique $\mathrm{n}^{\circ} 105$ juillet $2014 \bullet 5$ 
la couleur du sang, du coquelicot, du rubis, etc. (extrémité du spectre solaire)» (Définition du Petit Robert pour l'adjectif rouge).

Ce double adossement aux réalités dénommées et à la science contemporaine explique que, depuis l'étude célèbre de Berlin et Kay (1969), ces termes aient été et soient encore le fer de lance de la sémantique cognitive référentielle universaliste. Selon ces auteurs, il existe un nombre restreint de termes fondamentaux (onze) ${ }^{1}$, ou "de base», organisés autour de valeurs saillantes du spectre, toujours les mêmes, qui s'opposent à des termes moins fondamentaux et dérivés : structure universelle, universaux chromatiques en somme, que l'on retrouve à l'œuvre aussi bien en arrière-plan de la structure de toutes les langues que dans les mécanismes de l'acquisition des langues (les termes de base en premier, les autres ensuite et seulement le cas échéant).

Cependant, c'est un fait connu que les cultures évoluent d'une manière qu'on ne peut pas ramener à des mécanismes cognitifs : on sait qu'Aristote, l'un des premiers théoriciens de l'arc-en-ciel, lui voit trois couleurs que l'on traduit en français par violet, vert et rouge - c'est-à-dire les extrémités du spectre plus une valeur centrale, mais pas une valeur de plus (Les Météorologiques, L. III, chap. 4, § 24). Et c'est un fait aussi qu'elles varient : non seulement la plupart des langues africaines, si on y cherche des couleurs, n'en distinguent pas plus de quatre (généralement autour du brun, du rouge, du jaune, du blanc), mais encore il semble bien, au-delà de cet exotisme chromatique, que la notion même de couleur leur soit étrangère :

Dans la plupart des civilisations d'Afrique, par exemple, peu d'importance est attachée à la frontière qui peut séparer la gamme des tons rouges de celle des bruns ou des jaunes, voire de celle des verts. En revanche, devant une couleur donnée, il est essentiel de savoir s'il s'agit d'une couleur sèche ou d'une couleur humide, d'une couleur tendre ou d'une couleur dure, d'une couleur lisse ou d'une couleur rugueuse, d'une couleur sourde ou d'une couleur sonore, parfois d'une couleur gaie ou d'une couleur triste. La couleur n'est pas une chose en soi. ${ }^{2}$

Il est par conséquent normal que, sur ce terrain aux enjeux à la fois sémantiques et philosophiques, les adversaires de l'universalisme perceptuel et cognitif aient aussi leurs thèses. François Rastier (1991) décrit rapidement les positions du débat : l'hypothèse dite de Sapir-Whorf garde des émules dans les sémantiques «relativistes», plus culturalistes, plus structurales ou poststructurales, moins cognitivistes?

En fin de compte, si nul désormais ne peut plus faire état d'un total arbitraire des lexiques des termes de couleur par rapport aux structures mentales

1. Red, orange, yellow, green, blue, purple, pink, brown, grey, black, white.

2. Pastoureau, 1989. Voir déjà Tornay, 1978, pour l'ancrage intrinsèquement culturel et tout relatif de la catégorie même de couleur.

3. On peut consulter par exemple le volume $X X$ n $^{\circ} 4$ de la revue Ethnologie française (1990). 
et à l'organisation du monde, nul ne peut non plus négliger la diversité fondamentale des catégorisations chromatiques de la réalité, tant sur le plan des langues que des discours.

L'analyse des significations culturelles des couleurs et des termes de couleur n'est pas impossible, mais très délicate. S'intriquent en effet les pratiques des couleurs, les valeurs des couleurs elles-mêmes et enfin le sens des termes de couleur. La pratique imprévisible des couleurs demande une observation et un constat. Ainsi les usages concrets du rouge supposent-ils une norme selon laquelle il sied mieux aux Ferrari qu'aux Porsche, s'emploie pour les sols plus facilement que pour les murs, etc. Quant auxvaleurs des couleurs, qui prennent une sorte d'indépendance par rapport à leurs pratiques, elles ne sont jamais unifiées, ainsi que le rappelle Pastoureau : danger du rouge des baies non comestibles, du camion des pompiers, du téléphone rouge; mais aussi passion des muqueuses turgescentes, des lèvres fardées, des roses pourpres; plaisirs encore des rideaux de théâtre où le rouge s'allie à l'or, ou plaisirs gustatifs des fraises et cerises. Le registre sémantique enfin révèle sous l'unité chromatique des spécifications particulièrement hétéroclites : communiste, congestionné et rubicond figurent ainsi parmi les 67 synonymes de rouge que répertorie le dictionnaire du Crisco 4.

Ainsi, la valeur des termes de couleur en discours s'alimente-t-elle à des sources très hétérogènes, où il faut distinguer des considérations de type cognitivo-perceptuel (couleurs plus ou moins «stimulantes» ou « saillantes», «chaudes»vs «froides») 5 aussi bien que socio-historiques (ex. du rouge «communiste»). Ici, plus encore qu'ailleurs, s’imposent donc des études sur des corpus définissant avec précision leur domaine d'investigation.

Le terrain politique est naturellement un poste d'observation privilégié : les couleurs des drapeaux sont nationales (pour la France, le tricolore, voire le " cocardier», dessinent une partie de l'identité nationale ${ }^{6}$; on se rappelle par ailleurs que Barthes (1964) a décrit dans l'alliance blanc/rouge/vert l'un des signifiants de ce qu'il a appelé l'«italianité »), couleurs «politiques» dessinant de manière transnationale comme un spectre d'opinions récupérant les valeurs diffuses de ces termes dont la signification dépasse de loin la simple description du visible.

Il est par ailleurs certain que le lexème couleur renvoie, dans la langue française, à l'opinion personnelle, ainsi que l'indique l'expression proverbiale «Des goûts et des couleurs on ne peut pas discuter». La forte dimension symbolique et d'affichage du chromatisme est nettement présente dans

4. http://www.crisco.unicaen.fr/des/synonymes/rouge. Consulté le 9 avril 2014.

5. Opposition dont on sait par ailleurs qu'elle est historique (ce n'est qu'à partir du $17^{\mathrm{e}}$ siècle que le blanc a lentement commencé à être considéré comme une couleur froide, écrit Pastoureau dans sa préface à Envies de blanc de Georges-François Rey, Albin Michel 2006).

6. Ils sont ainsi des «emblèmes de la France » : Pastoureau, 1998. 
des expressions lexicalisées qui transcrivent les sèmes des vocables couleurs nationales, couleurs républicaines, à tel point que prendre couleur renvoie à l'acte de s'encarter, à l'appartenance politique, et qu'à l'inverse, les opinions incolores sont une neutralité politique de mauvais aloi, sorte d'absence de conscience politique personnelle plutôt que sens aigu de l'équilibre.

\section{Analyse des discours politiques}

La référence aux partis politiques au moyen de leur couleur emblématique semble être une pratique courante du discours politique et journalistique. Les adjectifs de couleur, utilisés comme tels (la tornade rose, c'est-à-dire des résultats d'élection en faveur du PS), comme substantifs (les Verts) ou comme adverbes (voter rouge, J.-P. Sartre dans Le sursis) ponctuent l'histoire politique des deux derniers siècles (pour en rester au rouge : Armée Rouge, l'Affiche rouge, Garde rouge, Brigades rouges, les rouges et les blancs de la révolution russe, les rouges et les bleus de la guerre d'Espagne, etc.).

Cette pratique autorise de nombreux retournements (voir l'histoire du rouge communiste, originellement destiné à signifier l'interdiction d'attroupement, qui deviendra l'emblème du peuple opprimé et de la révolution en marche), détournements, réappropriations (« rose » est au départ un quolibet que la SFIO lançait contre les radicaux, rouges dévoyés), l'humour (les éléphants roses du PS), la réfection de clichés (marée verte, péril rouge, Khmers roses, Khmers verts, bleu Marine), de phénomènes métonymiques (le Petit Livre rouge, dont le véritable nom est Citations du président Mao Tse Toung, le vert-de-gris pour désigner les néonazis, et plus récemment, les Bonnets rouges), ces procédés pouvant être associés au jeu sur le signifiant ou même le signifié métonymique (la rose du PS), ou à une forme complexe d'iconicité (les Verts) jusqu'à des jeux de mots - «On sait ce que donne l'alliance du rouge et du vert... » (« Le noir et le rouge », Le Point, 22 février 1982, à propos de la rencontre entre Michel Noir et Daniel Cohn Bendit), le Rassemblement bleu Marine - et à diverses formes de sloganisation ("Better dead than red", «Black is beautiful»).

Mais bien au-delà du jeu littéraire et de la recherche du slogan, les valeurs que symbolisent les couleurs sont fortement ancrées culturellement et peuvent faire de l'utilisation de l'adjectif de couleur un outil argumentatif fort, comme l'indiquent les discussions et débats médiatiques sur une société black-blancbeur qui aurait remplacé la civilisation bleu-blanc-rouge au moment de la coupe du monde de football de 1998, durant laquelle il a été dit qu'il ne fallait pas laisser le monopole du bleu-blanc-rouge au Front national. Ce fait est d'autant plus important que les langues sont émaillées de locutions comprenant des adjectifs de couleur et que les valeurs symboliques - qu'il s'agisse de valeurs culturelles (rouge de la passion, de l'interdit, de la violence, du plai- 
sir, du danger, de l'égalité) ou politiques (noir du nazisme, de l'anarchie, de l'Église) - sont empreintes d'une polyvalence qui ouvre une large voie aux jeux connotatifs les plus variés.

Il s'agira donc d'étudier la référence à la couleur dans le discours politique et son incidence sur l'argumentation, que ces valeurs soient plus ou moins stabilisées ou au contraire «en mouvement». Si le cognitivo-perceptuel compose avec le sociohistorique dans la catégorisation chromatique du visible et brouille les cartes dans la bataille anthropologique entre universalistes et relativistes, l'usage politique de la couleur fait résolument pencher la balance du côté du primat des facteurs socio-historiques.

\section{Composition du dossier}

Classées chronologiquement afin de montrer la permanence de questions de chromatisme politique, les contributions présentées sont tant diachroniques et historiques que comparatistes et synchroniques. Ce dossier, dont l'objectif est de dépasser l'ici et maintenant des couleurs politiques, propose un spectre des grandes directions de réflexion traversant cette problématique.

- Les «spectres politiques» et chromatiques nationaux en diachronie et en synchronie :

Aude Gerbaud-Dontenwille trace l'histoire de l'expression la ligne bleue des Vosges, employée en 1893 par Jules Ferry dans son testament et qui connaît un développement formulaire jusqu'en 1920 afin de renforcer le trait national. Par le truchement de l'étude de cette expression, cet article analyse le rapport particulier de l'histoire de France à la couleur bleue, revendiquée et par les partisans d'une monarchie chrétienne, et par une droite néonationaliste comme par les héritiers de la Grande Révolution.

- Une géopolitique des couleurs (nationales vs interculturelles : les Verts / die Grünen) :

Laurence Vignes effectue une étude onomastique du nom de parti politique Les Verts qu'elle complète par celle de la symbolique identitaire des couleurs qui le représentent. Considérant que ces deux systèmes sémiotiques se complètent, elle analyse les différentes organisations concurrentes dans le champ de l'écologie politique à travers le monde. Son article montre que vert s'est imposé au détriment d'écologie/écologiste, succès à attribuer à l'efficacité pragmatique de l'utilisation d'une couleur comme nom. Reprenant Pastoureau (2013), elle souligne que le vert est devenu davantage une idéologie qu'une couleur, à l'instar du rouge il y a quelques décennies.

- Les formes de motivation du «sens politique de la couleur» - métonymiques, métaphoriques, extension-spécialisation (bleu-blanc-rouge / «tricolore»)... et leur relation à l'histoire sociopolitique (nationale, internationale) : 
Camille Biros propose une étude du rôle des termes de couleur dans le discours environnemental. S'appuyant sur une observation de la communication des organisations au Royaume-Uni de 2000 à 2012, son article interroge la valeur symbolique de la couleur verte et décrit la richesse des phénomènes discursifs engendrés par la place centrale de l'adjectif green dans ces diverses productions discursives. Y est constaté le fort ancrage culturel des valeurs associées à différentes teintes, qui explique la forte présence du vert dans ce type de discours. Cependant, le discours de l'écologie évolue au gré des découvertes scientifiques qui déplacent le centre des préoccupations vers différentes questions environnementales. Actuellement, c'est le bleu qui est convoqué, mais toujours sur un fond de vert, se différenciant de cette couleur et ceci à diverses fins.

- Les usages polémiques de la couleur: couleurs revendiquées, déniées, attribuées, réappropriées, détournées, disputées (black par les Afro-Américains)7 :

S'appuyant sur un corpus constitué de différents outils de communication politique, Christine Chevret-Castellani retrace la dialectique du noir et de la transparence du Parti pirate français lors de la campagne électorale législative de 2012. L'article démontre la différence entre le discours visuel et le discours verbal, différence que son auteur affirme révéler une contradiction performative entre l'énonciation visuelle et l'énonciation verbale. De fait, le discours de la transparence construit par le Parti pirate est celui du crypto-anarchisme, au sens où le transparent n'est pas utilisé au sens figuré de «qui permet la possibilité totale de contrôle » mais au sens informatique de «qui n'affecte pas le contenu du message », rendant certains éléments de code invisibles à l'utilisateur. Ainsi, le noir, emprunté à la culture pirate, n'entre pas en contradiction avec le transparent, issu de la culture hacker. Cependant, la campagne pour les élections législatives place cette formation politique face à un dilemme communicationnel qu'elle résout par un lissage sémiotique dans une optique de légitimation.

- La couleur orange comme signe, puis comme scène politique :

Valentyna Dymytrova a analysé les usages de l'adjectif de couleur orange et de ses dérivés lors de la révolution du même nom dans un corpus de presse français et ukrainien, mais aussi dans les discours politiques et journalistiques de ce pays. Cette analyse, qui porte sur trois langues (français, ukrainien et russe), vise à démontrer que les couleurs expriment d'une façon métaphorique la conflictualité inhérente à la logique du politique, reposant sur une confrontation avec l'identité d'autres acteurs par opposition, à la fois réelle et symbolique (Lamizet, 2002). La façon de désigner une couleur peut ainsi devenir un marqueur important des rapports de pouvoir et un moyen d'expression de l'opinion qui permet d'ancrer une identité en politique.

7. Voir Bonnet, 2011. 


\section{Bibliographie indicative}

BARTHes Roland, 1964, «Rhétorique de l'image», Communication, n² 4, p.41-42.

BERLIN Brent, KAY Paul, 1969, Basic Color Terms. Their Universality and Evolution, Berkeley, University of California Press.

Bonnet Valérie, 2011, “'Don’t call me Nigger, Whitey”. L'autodésignation de la communauté afro-américaine et la construction identitaire», Communication, vol.XXVIII, $n^{0} 2$, mis en ligne le 27 juillet 2011, consulté le 5 septembre 2011 : http://communication.revues.org/index1803.html.

DuboIS Danièle, CANCE Caroline, 2009, "Mettre un terme aux couleurs de base. Déconstruction d'un paradigme dominant», Le Sentir et le Dire. Concepts et méthodes en psychologie et linguistique cognitives, D. Dubois éd., Paris, L'Harmat$\tan$, p. 75-100.

DuboIs Danièle, GrineVALD Colette, 1999, «Pratiques de la couleur et dénominations », Faits de langues, $\mathrm{n}^{\circ} 14, \mathrm{p} .11-25$.

LAMIZET Bernard, 2002, Politique et identité, Lille, Presses universitaires de Lille.

Leduc-Adine Jean-Pierre, 1980, "Polysémie des adjectifs de couleur», Cahiers de lexicologie, vol.XXXVII, p. 67-90.

NDIAYE Pap, 2006, "Questions de couleur. Histoire, idéologie et pratiques du colorisme », De la question sociale à la question raciale? Représenter la société française, É. Fassin, D. Fassin éd., Paris, La Découverte, p. 37-54.

Pastoureau Michel, 1989, Couleurs, images, symboles, Paris, Léopard d'or.

- 1998, Les emblèmes de la France, Paris, Bonneton.

- 2003, Les couleurs de notre temps, Paris, Bonneton.

- 2010, Couleurs, Paris, Chêne.

- 2013, Vert. Histoire d'une couleur, Paris, Le Seuil.

RASTIER François, 1987, Sémantique interprétative, Paris, Presses universitaires de France.

- 1991, Sémantique et recherches cognitives, Paris, Presses universitaires de France.

ToRnAYSerge éd., 1978, Voir et nommerles couleurs, Nanterre, Laboratoire d'ethnologie et de sociologie comparative.

WierzbicKA Anna, 2006, "Sens et grammaire universelle : théorie et constats empiriques», Linx, $\mathrm{n}^{\circ} 54$, mis en ligne le $1^{\mathrm{er}}$ août 2007, consulté le 11 juin 2012 (http:// linx.revues.org/520); DOI : 10.4000/linx.520. 